\title{
Synthesis and Evaluation of the Bis-Nor-Anachelin Chromophore as Potential Cyanobacterial Ligand
}

\author{
Yann Bethuel and Karl Gademann* \\ Laboratorium für Organische Chemie der ETH Zürich, \\ HCI G 337, CH-8093 Zürich, Switzerland \\ gademann@org.chem.ethz.ch
}

Table of contents:

S2: Experimental part: general methods

S3: Experimental part precursor compound 4

S4: Experimental part compound 4

S5: Figure 6, figure 7

S6: Figure 8, figure 9

S7: ${ }^{1} \mathrm{H}$ NMR compound 4

S8: ${ }^{1} \mathrm{H}$ NMR compound 5

S9: ${ }^{1} \mathrm{H}$ NMR compound 6

S10: ${ }^{1} \mathrm{H}$ NMR compound 7

S11: ${ }^{1} \mathrm{H}$ NMR compound 8

S12: ${ }^{1} \mathrm{H}$ NMR compound 9

S13: ${ }^{1} \mathrm{H}$ NMR compound $\mathbf{1 0}$

S14: MS compound 10 


\section{Experimental.}

General. Boc-Ser(OBn)-OH was purchased from Senn Chemicals, Switzerland. Solvents for work-up and chromatography were distilled from technical quality. Solvents used for chemical transformations were either puriss. quality or dried over columns of dry aluminum oxide. The preparation of nitro-DOPA derivative 4 following a modified procedure of Kolosa and Miller ${ }^{1}$ is reported in the Supporting Information.

[1] Kolasa, T.; Miller, M. J. J. Org. Chem. 1990, 55, 4246-4255. 


\section{(2S)-2-allyloxycarbonylamino-3-(3,4-dimethoxy-phenyl)-propionic acid ethyl ester}

To a suspension of $L$-DOPA $(11 \mathrm{~g}, 0.056 \mathrm{~mol})$ in $\mathrm{MeOH}(200 \mathrm{~mL})$ cooled at $-40^{\circ} \mathrm{C}$ was added thionyl chloride $(9.32 \mathrm{~g}, 0.078 \mathrm{~mol})$ dropwise over 1.5 hours. After 1 day at RT the reaction was complete and $\mathrm{MeOH}$ was removed under reduced pressure. To the residue suspended in $\mathrm{H}_{2} \mathrm{O}$ $(100 \mathrm{~mL})$, were added subsequently $\mathrm{NaHCO}_{3}(9.41 \mathrm{~g}, 0.112 \mathrm{~mol})$ and allylchloroformiate (8.08 $\mathrm{g}, 0.067 \mathrm{~mol})$ in THF $(100 \mathrm{~mL})$. The reaction mixture was then stirred over night at room temperature. The solvent was evaporated and the aqueous solution was extracted $3 \mathrm{x}$ with AcOEt. The combined organic layers were dried over $\mathrm{Na}_{2} \mathrm{SO}_{4}$, filtered and evaporated under reduced pressure. To the residue dissolved in absolute ethanol $(350 \mathrm{~mL})$ was added anhydrous $\mathrm{K}_{2} \mathrm{CO}_{3}(16.96 \mathrm{~g}, 0.123 \mathrm{~mol}), \mathrm{MeI}(16.75 \mathrm{~g}, 0.118 \mathrm{~mol})$ and $\mathrm{NaI}(0.839 \mathrm{~g}, 0.0056 \mathrm{~mol})$. The mixture was heated to reflux for 3 hours. It was then poured into water $(1 \mathrm{~L})$. The solution was extracted with EtOAc and the combined organic phases were dried over $\mathrm{Na}_{2} \mathrm{SO}_{4}$, filtered and evaporated under reduced pressure. FC (AcOEt / Hexane 4:6) gave 3 (16 g, 0.0476 mol, 85\%). White solid. $\mathrm{M}_{\mathrm{p}}=63-65^{\circ} \mathrm{C} . \mathrm{R}_{\mathrm{f}}=0.54(\mathrm{AcOEt} / \mathrm{Hexane} 5: 5) .[\alpha]_{\mathrm{D}}=+37.0\left(\mathrm{C}=1.13, \mathrm{CH}_{2} \mathrm{Cl}_{2}, \mathrm{~T}=\right.$ $\left.26^{\circ} \mathrm{C}\right) .{ }^{1} \mathrm{H}-\mathrm{NMR}\left(\mathrm{CDCl}_{3}, 300 \mathrm{Mhz}\right) 1.08(\mathrm{t}, 3 \mathrm{H}, J=7.16), 2.84-2.92(\mathrm{~m}, 2 \mathrm{H}), 3.67(\mathrm{~s}, 6 \mathrm{H}), 4(\mathrm{q}$, $2 \mathrm{H}, J=7.16), 4.38(\mathrm{~d}, 2 \mathrm{H}, J=5.3), 4.4(\mathrm{~m}, 1 \mathrm{H}), 5.03\left(\mathrm{dd}, 2 \mathrm{H}, J_{1}=2.18, J_{2}=11.8\right), 5.47(\mathrm{bd}, 1 \mathrm{H}$, $J=8.4), 5.67-5.73(\mathrm{~m}, 1 \mathrm{H}), 6.53-6.55(\mathrm{~m}, 2 \mathrm{H}), 6.63(\mathrm{~d}, 1 \mathrm{H}, J=8.4) .{ }^{13} \mathrm{C}-\mathrm{NMR}\left(\mathrm{CDCl}_{3}, 75 \mathrm{MHz}\right)$ $14.1,37.6,54.9,55.61,55.66,61.2,65.5,111,112.2,117.3,121.2,128.2,132.5,147.8,148.6$, 155.34, 171.5. IR 3432m, 3021s, 2939m, 1720s, 1515s. MS $360.1\left(100,[\mathrm{M}+\mathrm{Na}]^{+}\right), 338.2(14$, $\left.[\mathrm{M}+\mathrm{H}]^{+}\right), 294.2\left(13,[\mathrm{M}-\mathrm{EtOH}]^{+}\right) . \mathrm{HRMS}$ calcd. For $\mathrm{C}_{17} \mathrm{H}_{23} \mathrm{NO}_{6} \mathrm{Na}(\mathrm{M}+\mathrm{H})^{+}: 360.1417$ found: 360.1410. 


\section{(2S)-2-allyloxycarbonylamino-3-(4,5-dimethoxy-2-nitro-phenyl)-propionic acid ethyl ester}

A solution of $3(4.73 \mathrm{~g}, 14 \mathrm{mmol})$ in $\mathrm{AcOH}(30 \mathrm{~mL})$ was cooled to $0^{\circ} \mathrm{C}$ and nitric acid $(10 \mathrm{~mL})$ was then added dropwise. The orange mixture was stirred at RT for 2 hours and poured into water $(150 \mathrm{~mL})$. The mixture was filtered, the solvent removed under redued pressure, and dried under high vacuum to give 4 (3.86 g, $0.010 \mathrm{~mol}, 72 \%)$. Yellow solid. $\mathrm{Mp}=114-116^{\circ} \mathrm{C} . \mathrm{R}_{\mathrm{f}}=0.47$ $\left(\mathrm{CH}_{2} \mathrm{Cl}_{2} / \mathrm{MeOH} 99: 1\right) .[\alpha]_{\mathrm{D}}=6\left(\mathrm{C}=0.62, \mathrm{CH}_{2} \mathrm{Cl}_{2}, \mathrm{~T}=25^{\circ} \mathrm{C}\right) .{ }^{1} \mathrm{H}-\mathrm{NMR}\left(\mathrm{CDCl}_{3}, 300 \mathrm{Mhz}\right) 1.25$ $(\mathrm{t}, 3 \mathrm{H}, J=6.2), 3.32\left(\mathrm{dd}, 1 \mathrm{H}, J_{1}=8.7, J_{2}=13.7\right), 3.52\left(\mathrm{dd}, 1 \mathrm{H}, J_{1}=6, J_{2}=13.7\right), 3.9(\mathrm{~s}, 3 \mathrm{H})$, 4.12-4.26 (m, 2H), $4.49(\mathrm{~d}, 1 \mathrm{H}, J=5.3), 4.64-4.72(\mathrm{~m}, 1 \mathrm{H}), 5.16-5.27(\mathrm{~m}, 2 \mathrm{H}), 5.47(\mathrm{~d}, 1 \mathrm{H}, J=$ 8.71), 5.77-5.88 (m, 1H), $6.74(\mathrm{~s}, 1 \mathrm{H}), 7.6(\mathrm{~s}, 1 \mathrm{H}) .{ }^{13} \mathrm{C}-\mathrm{NMR}\left(\mathrm{CDCl}_{3}, 75 \mathrm{MHz}\right)$ 14.1, 35.9, 54.5, $56.3,56.4,61.8,65.8,108,113.7,117.8,126.6,152.8,171.2$. IR 3022m, 1721s, 1584w, 1524s, 1335m, 1272s. MS $405.1\left(100,[\mathrm{M}+\mathrm{Na}]^{+}\right), 389.1\left(43,[\mathrm{M}-\mathrm{EtOH}]^{+}\right)$. HRMS calcd. for $\mathrm{C}_{17} \mathrm{H}_{22} \mathrm{~N}_{2} \mathrm{O}_{8} \mathrm{Na}(\mathrm{M}+\mathrm{Na})^{+}:$405.1268, found: 405.1268. 
Figure 6: Air oxidation of compound $10(0.15 \mathrm{mM})$ at $\mathrm{pH}=9.2$ in degassed phosphate buffer (50 mM). UV-Vis spectra were measured every 30 seconds and air was added in between each measurement.

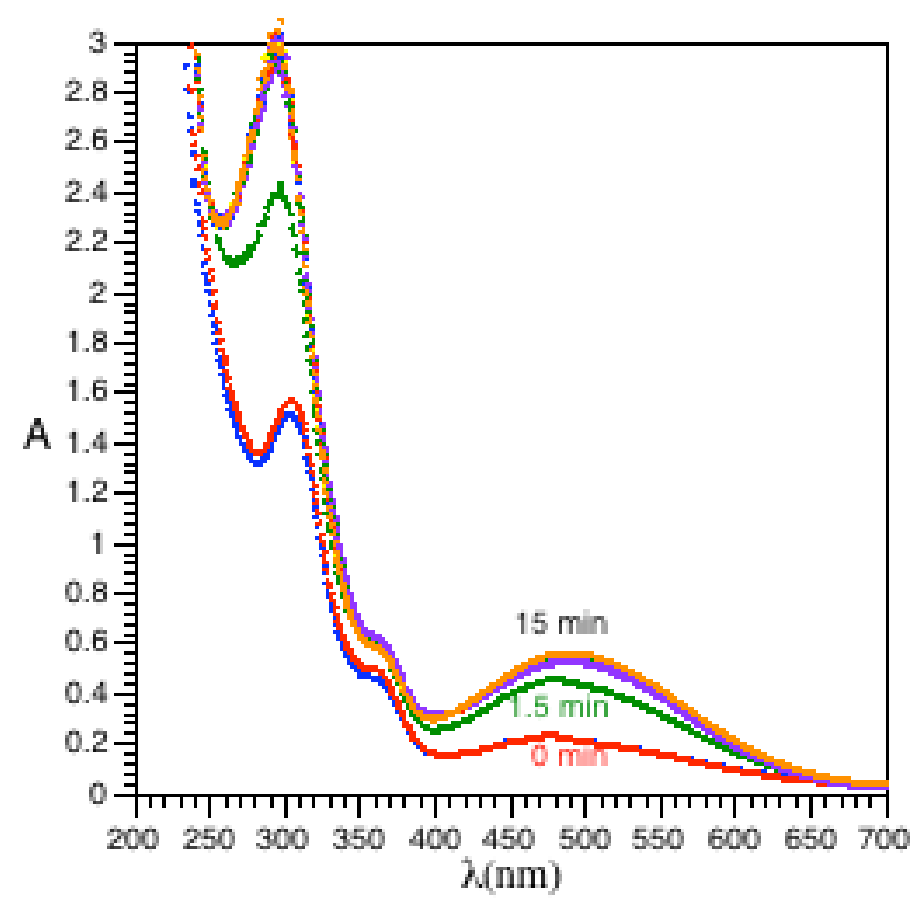

Figure 7: Oxidized form of compound $\mathbf{1 0}$ at $\mathrm{pH}=4,6.5,9.2$.

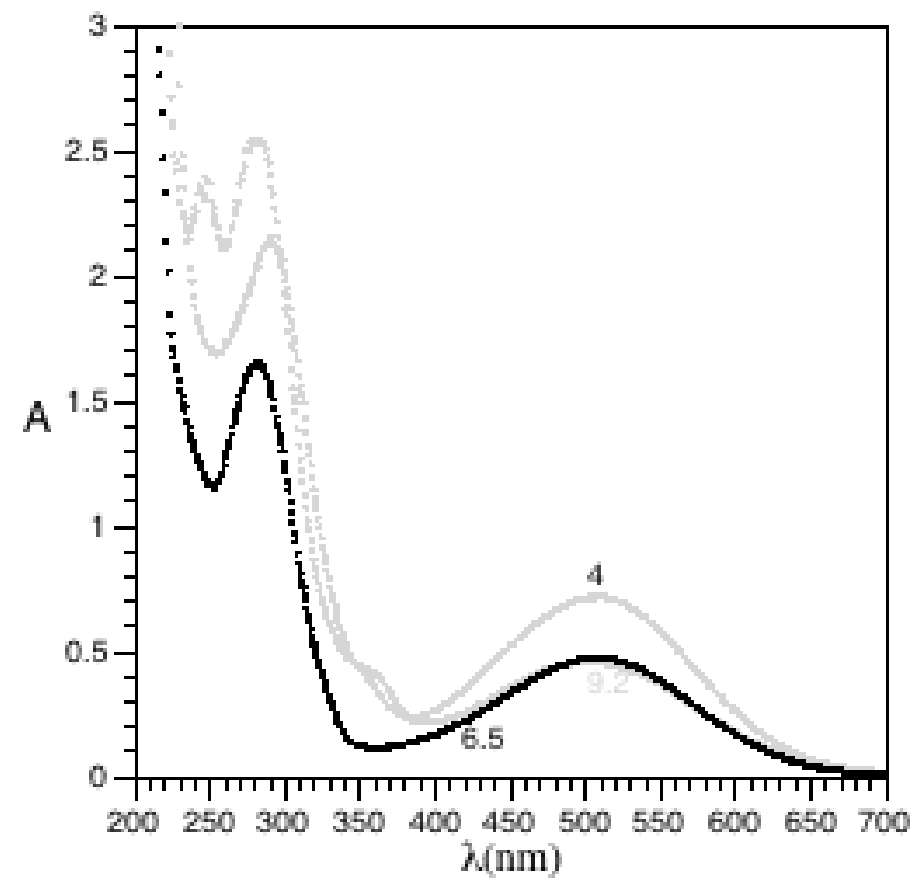


Figure 8: Kinetic of the mushroom tyrosinase oxidation of compound $\mathbf{1 0 .}$

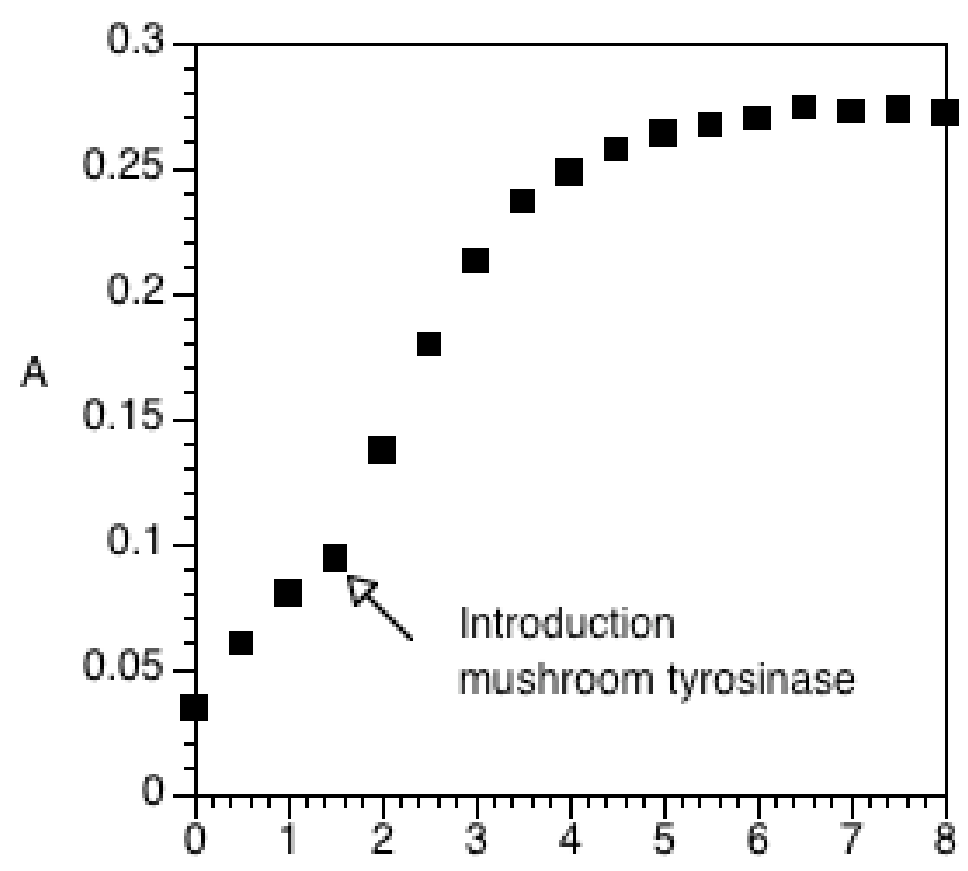

Figure 9: Oxidation of the bis-nor-anachelin chromophore 10 with $\mathrm{Fe}(\mathrm{III})$ at $\mathrm{pH}=4$.

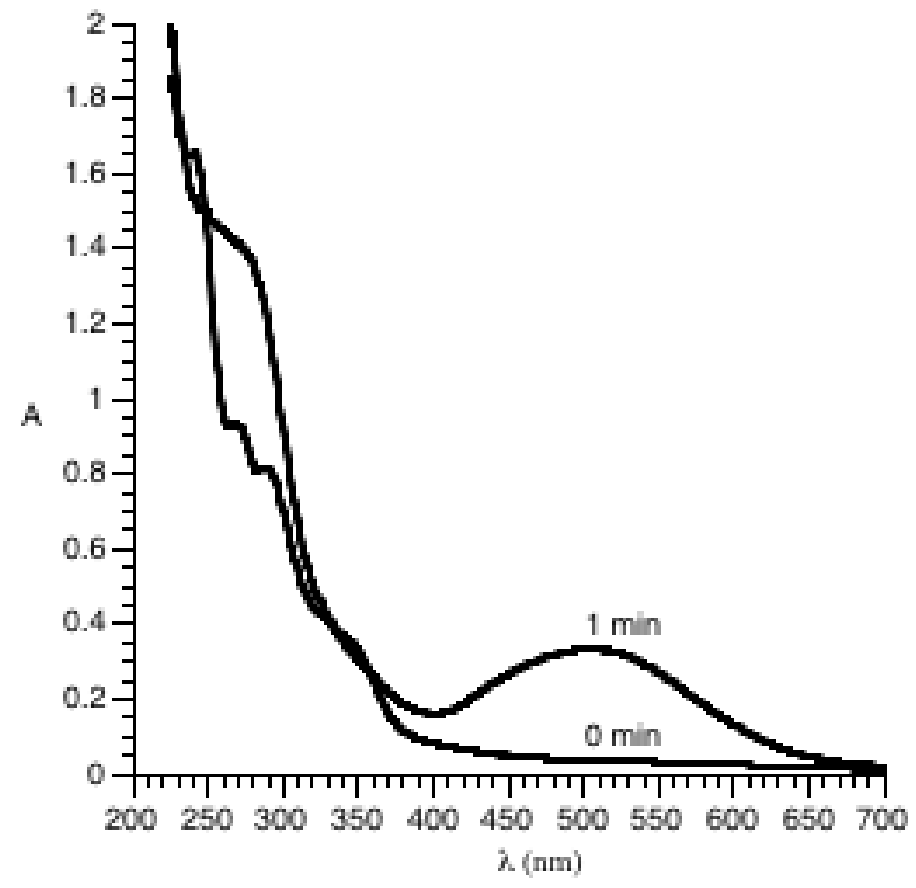




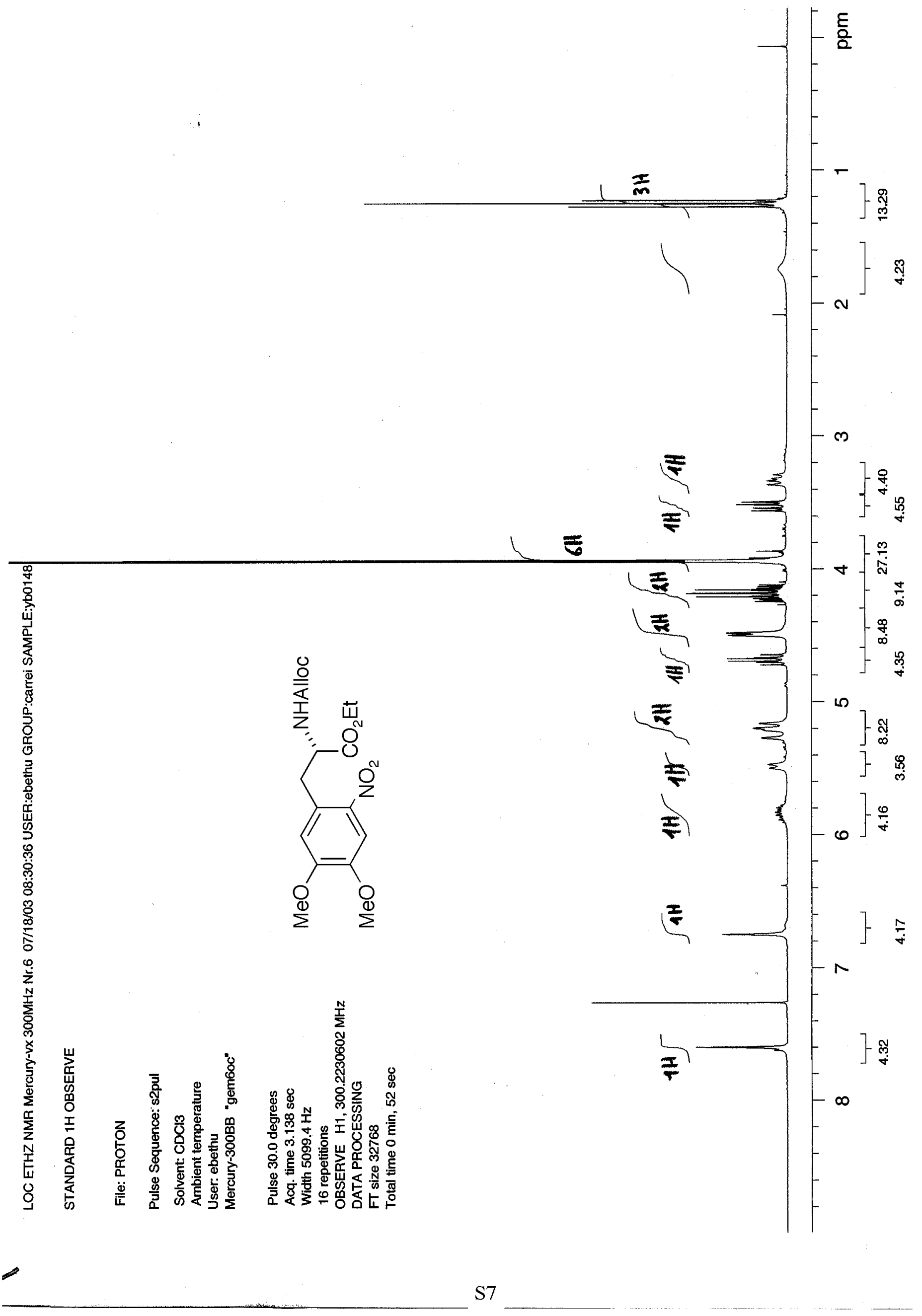




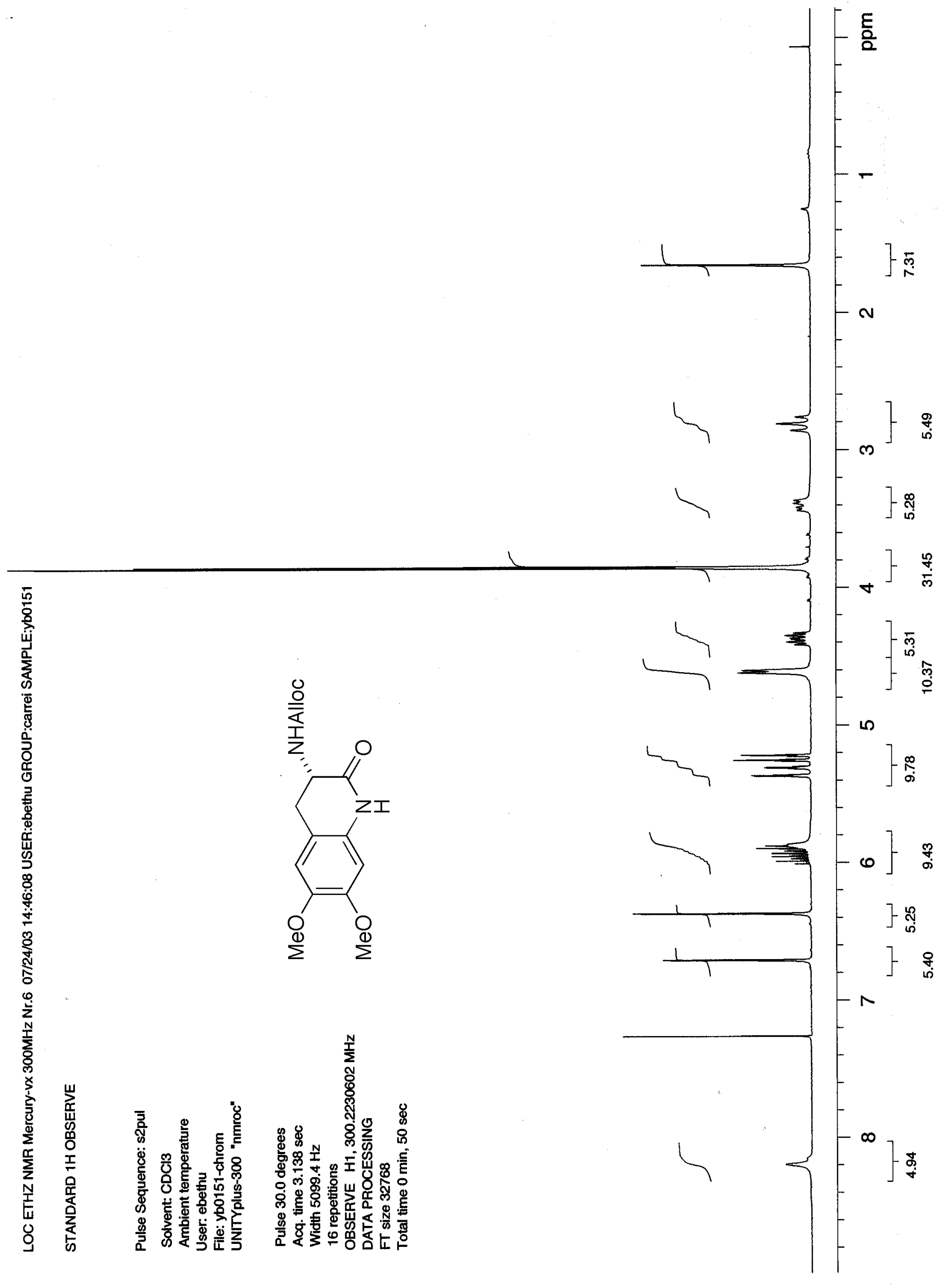




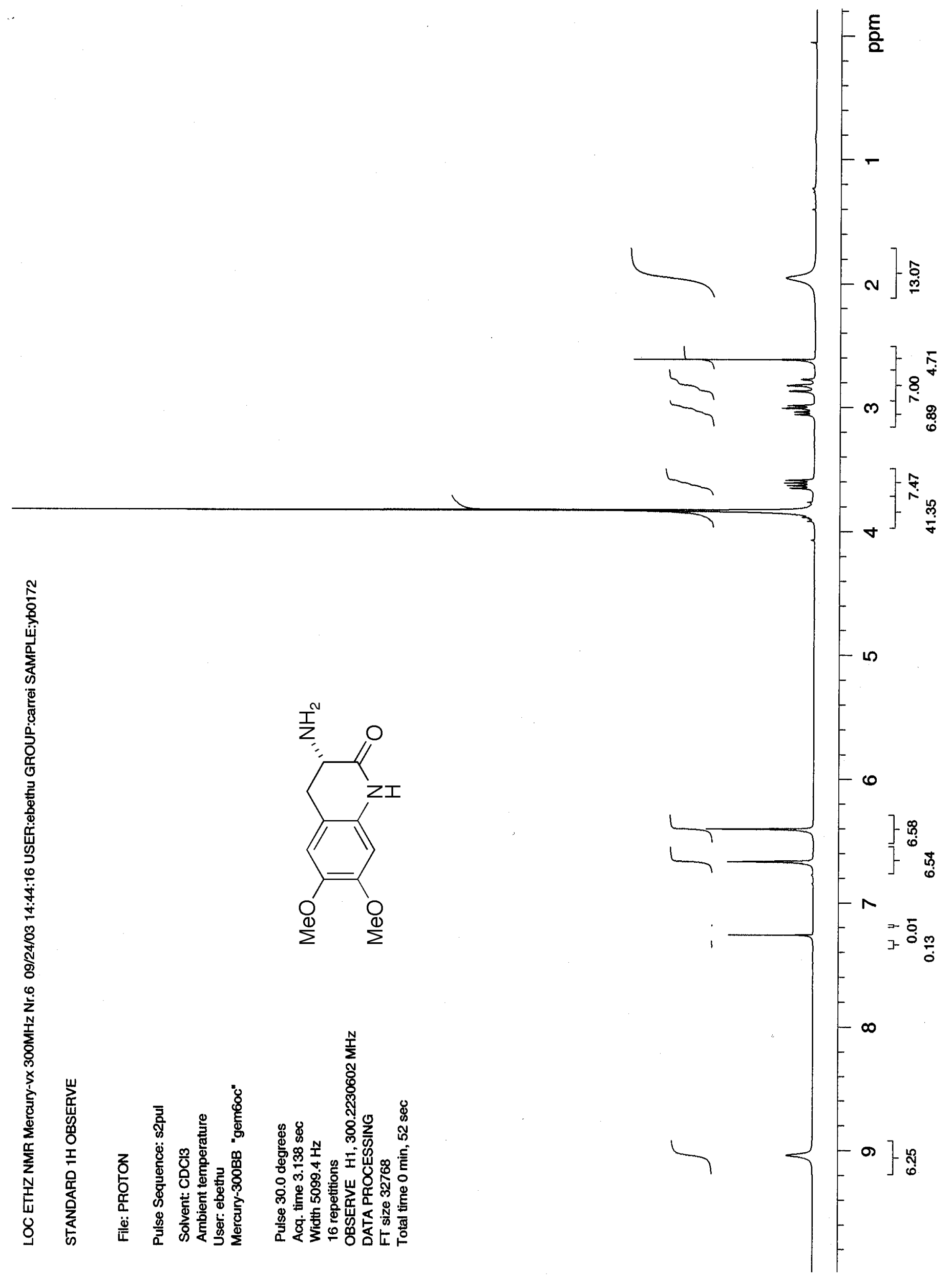




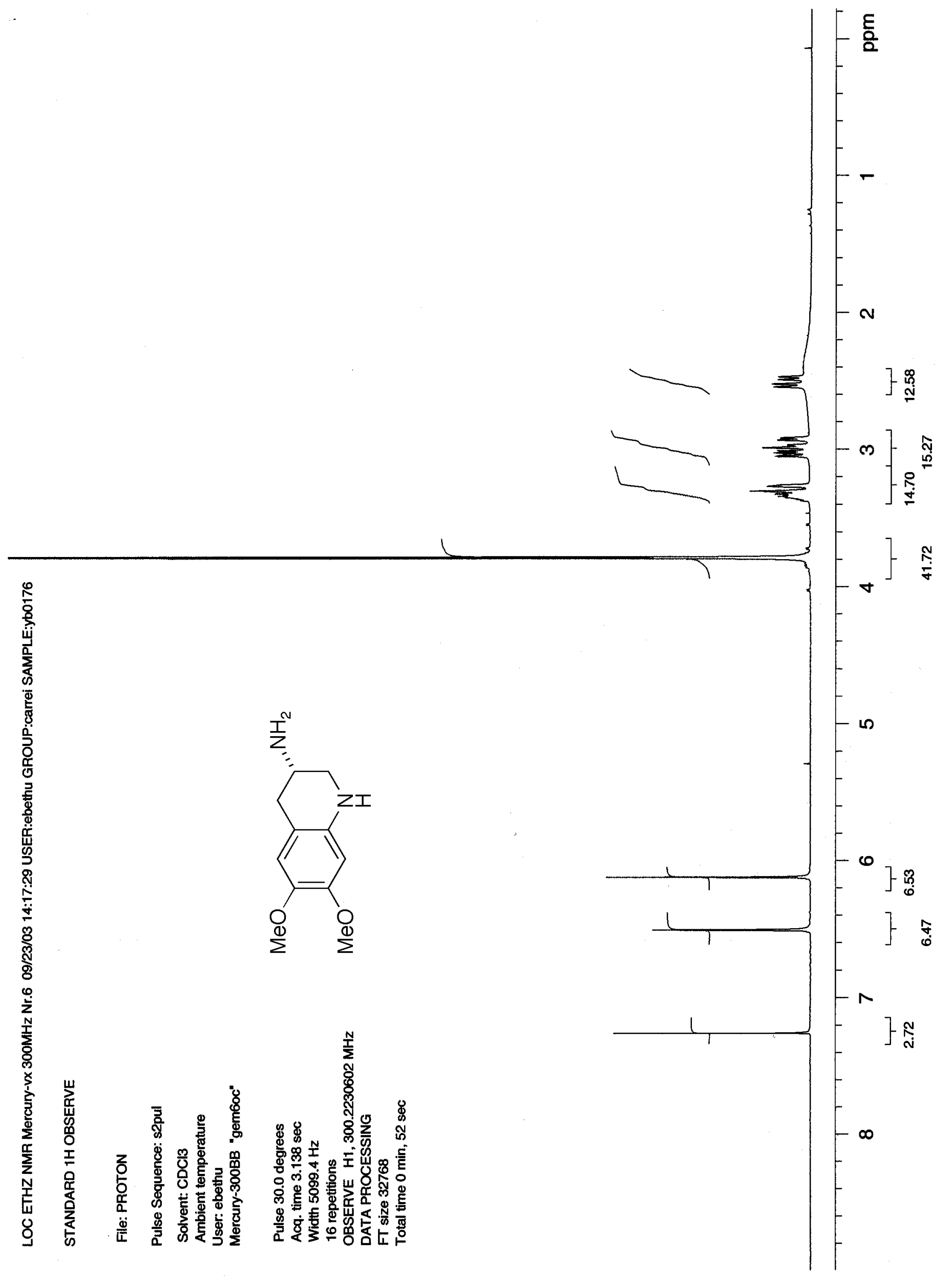




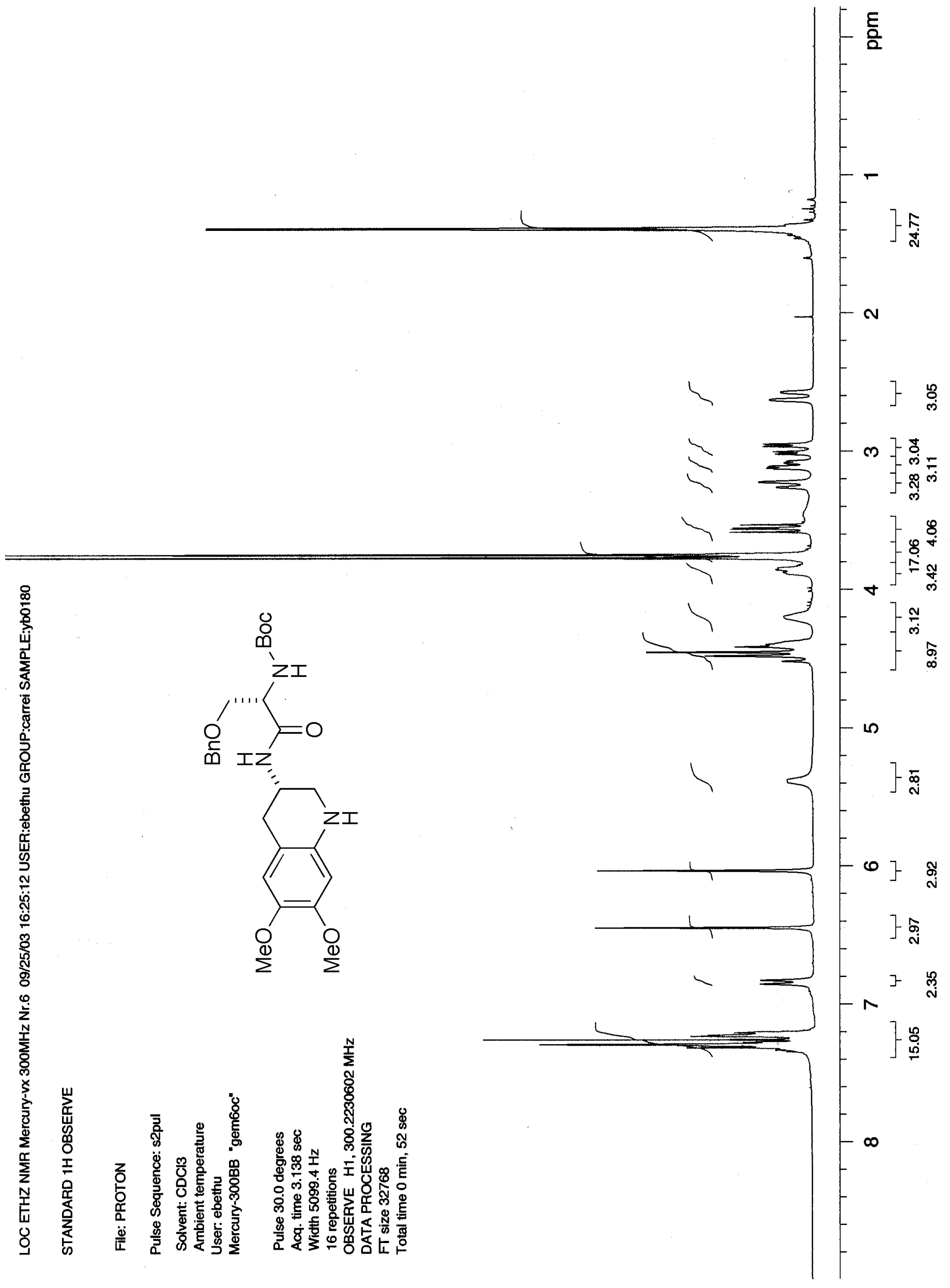




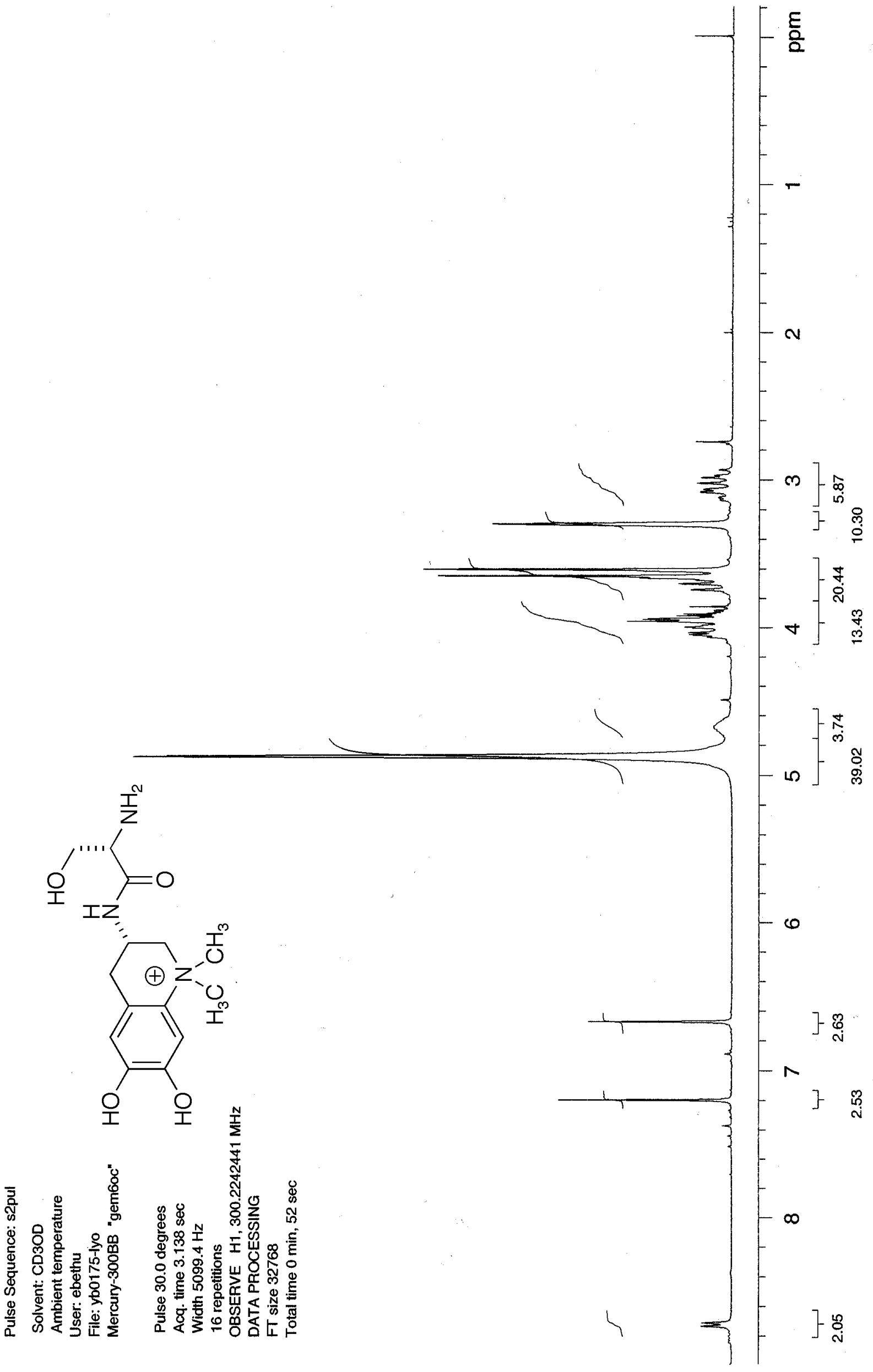




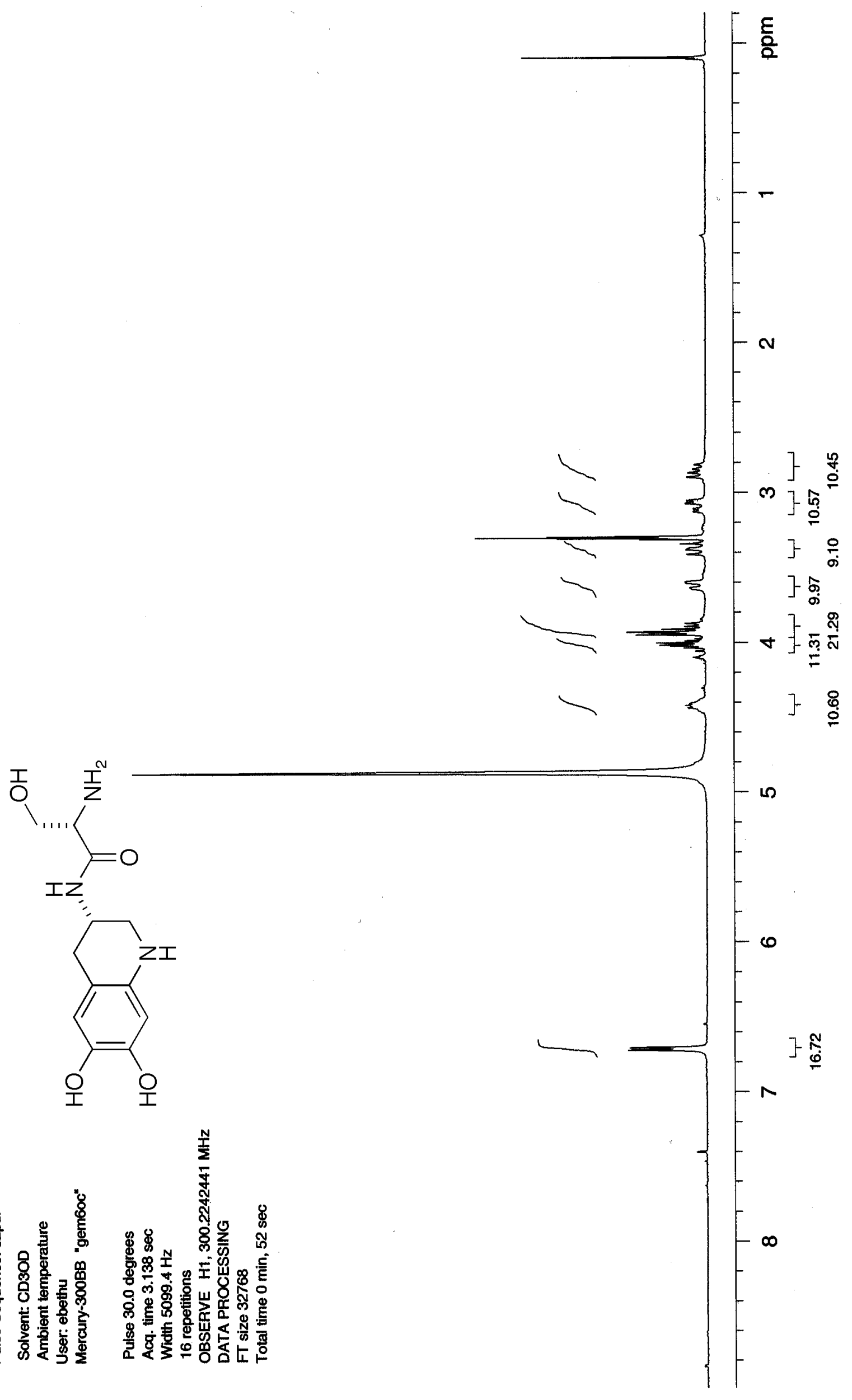


宇莫

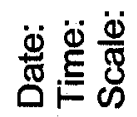

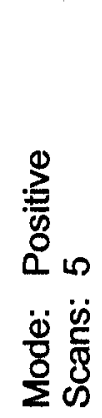

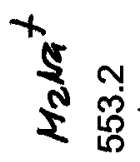

$\stackrel{6}{\circ}$

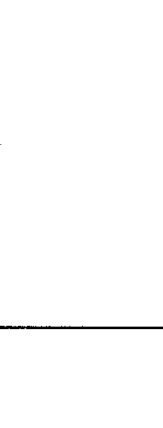

